\title{
Rapidly enlarged inflammatory hepatocellular adenoma: A case report
}

\author{
Kenji Koneri $^{1 \#}{ }^{1}$ Hidetaka Kurebayashi ${ }^{1}$, Katsuji Sawai ${ }^{1}$, Mitsuhiro Morikawa ${ }^{1}$, Makoto Murakami ${ }^{1}$, \\ Yasuo Hirono $^{1}$, Takanori Goi ${ }^{1}$, Atsushi Iida ${ }^{1}$, Kanji Katayama ${ }^{1}$, Hiroshi Itoh ${ }^{2}$, Motoko Sasaki ${ }^{3}$, \\ Yasuni Nakanuma ${ }^{3}$, Akio Yamaguchi ${ }^{1}$ \\ ${ }^{1}$ Department of Gastroenterological Surgery, University of Fukui, Fukui, Japan \\ ${ }^{2}$ Department of Tumor Pathology, University of Fukui, Fukui, Japan \\ ${ }^{3}$ Department of Human Pathology, Kanazawa University Graduate School of Medicine, Kanazawa, Japan \\ Email: ${ }^{*}$ koneri@hotmail.co.jp
}

Received 9 June 2013; revised 9 July 2013; accepted 24 July 2013

Copyright (C) 2013 Kenji Koneri et al. This is an open access article distributed under the Creative Commons Attribution License, which permits unrestricted use, distribution, and reproduction in any medium, provided the original work is properly cited.

\begin{abstract}
We present a rare case of rapidly enlarging inflamematory hepatocellular adenoma (IHCA) in a 60-yearold Japanese man. Screening abdominal computed tomography (CT) for the fatty liver patient revealed a $1.7-\mathrm{cm}$ liver mass in the anterior segment of the liver. After 19 months, the lesion had rapidly enlarged to 6 $\mathrm{cm}$ in diameter and the patient was referred to our hospital. On perflubutane microbubble contrast-enhanced ultrasonography, the tumor showed a characteristic centripetal filling pattern in the vascular phase. We performed hepatic anterior segment resection because we could not rule out malignant tumor. Histopathological examination showed hyperplasia of mildly atypical hepatocytes and sinusoidal dilatation with marked inflammatory cell infiltration. Immunohistological staining revealed positive staining for serum amyloid $A$ and $\mathrm{C}$-reactive protein; therefore, we diagnosed this tumor as IHCA. The patient remains alive $\mathbf{4 2}$ months after operation without evidence of recurrence.
\end{abstract}

Keywords: Inflammatory Hepatocellular Adenoma; Perflubutane Microbubble Contrast-Enhanced Ultrasonography; Surgery; Rapid Enlargement

\section{INTRODUCTION}

A new classification for hepatocellular adenoma (HCA)

"Author contributions: K. Koneri, H. Kurebayashi and A. Yamaguchi treated the patient and wrote the manuscript; T. Sawai, M. Morikawa, M. Murakami, Y. Hirono, T. Goi, A. Iida and K. Katayama treated the patient; H. Itoh, M. Sasaki and Y. Nakamura contributed to the pathological examination.

No support has been received from any company.

${ }^{\#}$ Corresponding author. was described in 2006 in the World Health Organization (WHO) classification of tumors of the digestive system. According to this classification, HCA is grouped into three subtypes based on genotype and phenotype as follows: hepatocyte nuclear factor (HNF) $1 \alpha$-inactivated HCA, $\beta$-catenin-activated HCA and inflammatory HCA (IHCA) [1-3]. Among these subtypes, IHCA results from a molecular gain-of-function mutation of the interleukin (IL)-6 pathway, and shows characteristic pathological features such as inflammatory cell infiltration, sinusoidal dilatation and increased expression of inflammatory proteins such as serum amyloid A (SAA) and C-reactive protein (CRP) [4]. A non-negligible number of IHCA patients have recently been reported in Western countries [2], but very few in Asian countries. We now report a case of an IHCA Asian male patient, who showed rapid enlargement of tumor and underwent surgical resection after preoperative perflubutane microbubble (Sonazoid, Daiichi-Sankyo, Tokyo, Japan) contrast-enhanced ultrasonography (Sonazoid-CEUS) and gadolinium ethoxybenzyl diethylenetriaminepentaacetic acid (Primovist, Bayer, Leverkusen, Germany) contrast-enhanced magnetic resonance imaging (Primovist-MRI).

\section{CASE REPORT}

The patient was followed by a local doctor for fatty liver and mild hypertension. In August 2007, a $1.7 \mathrm{~cm}$ tumor was detected in the anterior segment of the liver by abdominal computed tomography (CT) for screening of fatty liver. After diagnosis as nodular hyperplasia (FNH), follow-up observations were planned. In May 2009, follow-up CT revealed rapid enlargement of the tumor up to $6 \mathrm{~cm}$ in diameter, so the patient was referred to our hospital. The patient was a Japanese male who was 60 years 
old upon admission, and had no history of diabetes, blood transfusion, steroid use or heavy drinking. His height, weight and body mass index were $165 \mathrm{~cm}, 72 \mathrm{~kg}$ and $26.11 \mathrm{~kg} / \mathrm{m}^{2}$, respectively. Laboratory studies showed mildly elevated levels of aspartate aminotransferase (40 $\mathrm{IU} / \mathrm{L}$ ), alanine aminotransferase (49 IU/L) and alkaline phosphatase (629 IU/L), but white blood cell count, serum C-reactive protein and feasting blood glucose were normal. Carcinoembryonic antigen, feto protein and desgamma-carboxy prothrombin levels were also normal, and negative results were obtained for HBs antigen and HCV antibody.

Enhanced computed tomography (CT) in August 2007 showed an ill-defined $1.7 \mathrm{~cm}$ tumor in the anterior segment of the liver. In May 2009, plain CT revealed slightly low-density tumor and enhanced CT revealed diffuse enhancement at the arterial phase and homogeneous enhancement at the delayed phase. The tumor had rapidly enlarged over1 year and 7 months (Figure 1).

For qualitative diagnosis, we performed US study. Brightness-mode (B-mode) US showed a well-demarcated hypoechoic tumor. We performed CEUS using 0.5 $\mathrm{ml}$ of Sonazoid followed by $10 \mathrm{ml}$ saline flush through a peripheral vein. At 18 seconds (sec) after injection, rapid peripheral enhancement was shown. Subsequently, centripetal filling was shown at $20 \mathrm{sec}$ and complete filling at $28 \mathrm{sec}$. After 12 minutes (Kupffer phase), delayed wash out and peripheral rim of sustained enhancement were observed. Re-enhancement was observed after re-injecting $0.5 \mathrm{ml}$ of Sonazoid (Figure 2).

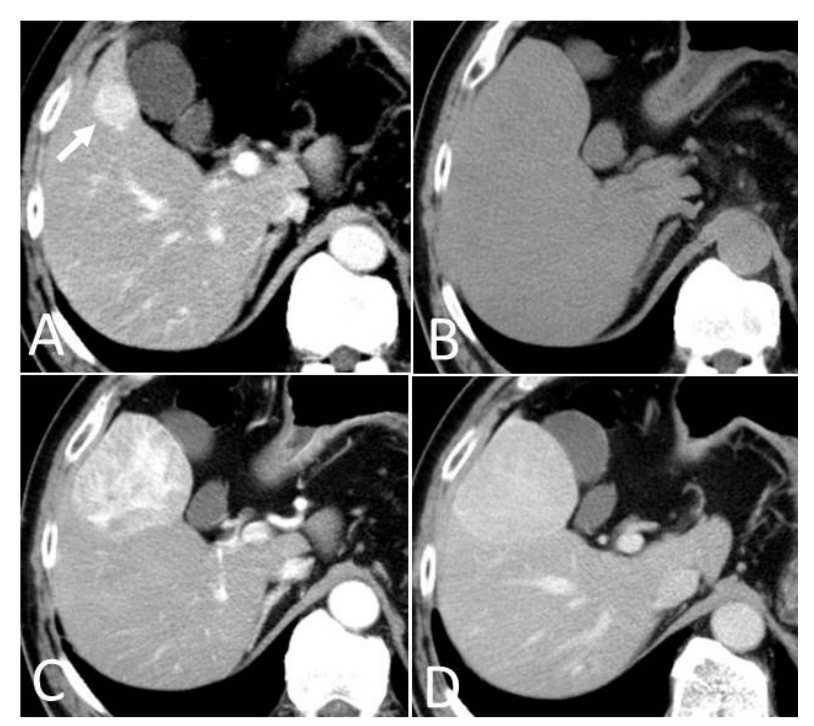

Figure 1. Enhanced computed tomography findings. (A) An ill-defined $1.7 \mathrm{~cm}$ tumor (arrow) was first detected in the anterior segment of the liver; (B) The tumor had rapidly enlarged to $6 \mathrm{~cm}$ after 1 year and 7 months. The lesion was slightly hypointense; (C) The entire tumor showed diffuse enhancement at the arterial-dominant phase; (D) The tumor showed uniform enhancement at the equilibrium phase.
Plain MRI showed slightly hyperintense tumor on T1and T2-weighted imaging, and hyperintensity on diffusion-weighted imaging. After enhancement using Primovist, diffuse enhancement was seen in the arterial phase. The enhancement effect continued in the portal phase. At 15 min after injection, in the hepatocyte phase, the tumor was hypointense compared with the background liver (Figure 3).

On the basis of these findings, hepatocellular adenoma was suspected, but we could not rule out the risk of rupture and malignant transformation. A biopsy was not considered because of a high risk of bleeding and dissemination of the tumor cells. Therefore we selected surgical resection. Intraoperative findings showed no ascites and no liver cirrhosis. The tumor was growing with he-

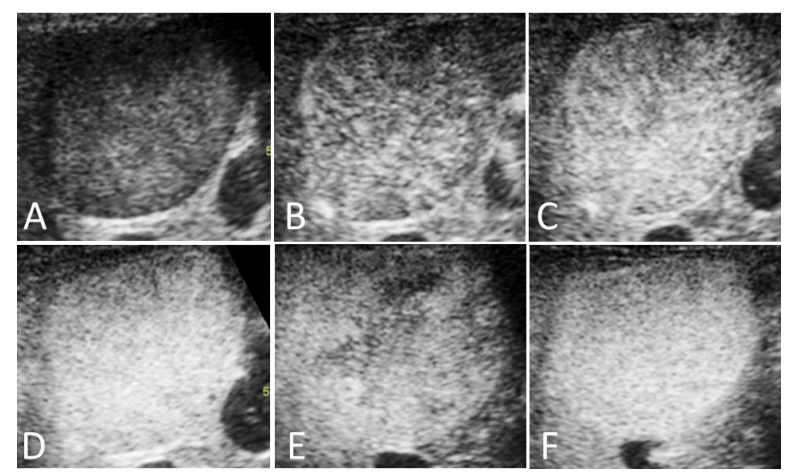

Figure 2. B-mode US and Sonazoid CEUS findings. (A) Welldemarcated hypoechoic mass was observed at B-mode; (B) The tumor showed initial peripheral enhancement at $18 \mathrm{sec}$ after Sonazoid injection; (C) The tumor showed centripetal filling at 20 sec; (D) The entire tumor showed complete filling at $28 \mathrm{sec}$; (E) Delayed washout and peripheral rim of sustained enhancement were observed in the Kupffer phase; (F) Strong re-enhancement was observed after re-injection of Sonazoid.

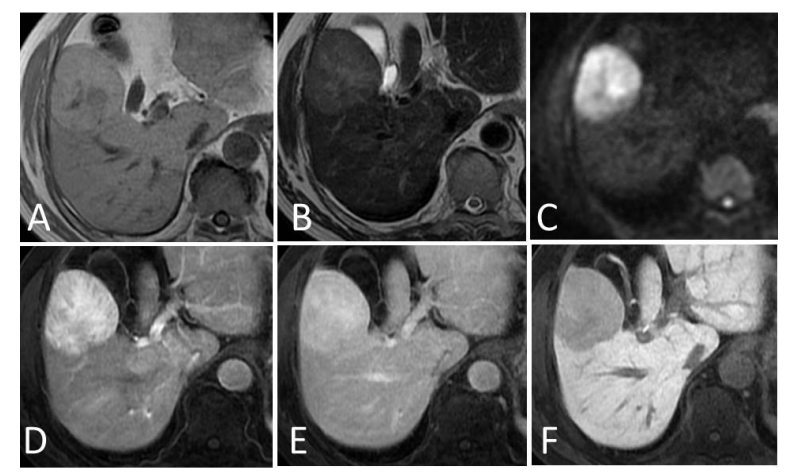

Figure 3. EOB-MRI findings. (A) The tumor was detected as slight hyperintensity on T1-weighted imaging; (B) The tumor showed slight hyperintensity on T2-weighted imaging; (C) Strong hyperintensity was evident on diffusion-weighted imaging; (D) After Primovist enhancement, diffuse strong enhancement was seen within the lesion in the arterial phase; (E) The entire tumor was enhanced in the portal phase; (F) The lesion was hypointense compared with the background in the hepatocyte phase. 
mispheric expansion from the liver surface and anterior segmentectomy was performed.

Macroscopically, the tumor was soft, the cut surface was brown and it was $6 \times 5 \mathrm{~cm}$ in size with a thin capsule. A dark purple congested area was evident at the center of the tumor. Histologically, hematoxylin and eosin (H\&E) staining revealed trabecular hyperplasia of mildly atypical hepatocytes and increased cell density, and there were marked sinusoidal dilatation and hemorrhage, thick-walled arteries and infiltration of inflammatory cells, which were predominantly lymphocytes (Figure 4). Immunohistological staining showed positive results for SAA and CRP (Figure 5). On the basis of these findings, we diagnosed the tumor as IHCA. The postoperative course was satisfactory, and the patient was discharged on day 16 after hepatectomy. The patient remains alive 42 months after operation, without evidence of recurrence.

\section{DISCUSSION}

Currently, HCA is classified into 3 subtypes based on genotype and phenotype: HNF $1 \alpha$-inactivated HCA, $\beta$-catenin-activated HCA and IHCA [1-3]. In Western countries, the incidence of HCA is $3-4$ per 1 million population and the "inflammatory" subtype accounts for approximately half of HCAs [3]. But the incidence of this subtype is exceptionally low in Asian countries, only 7 IHCA cases were reported by Sasaki from Japan [5].

Use of oral contraceptives, glycogen storage disease and anabolic hormone treatment such as steroid and an-

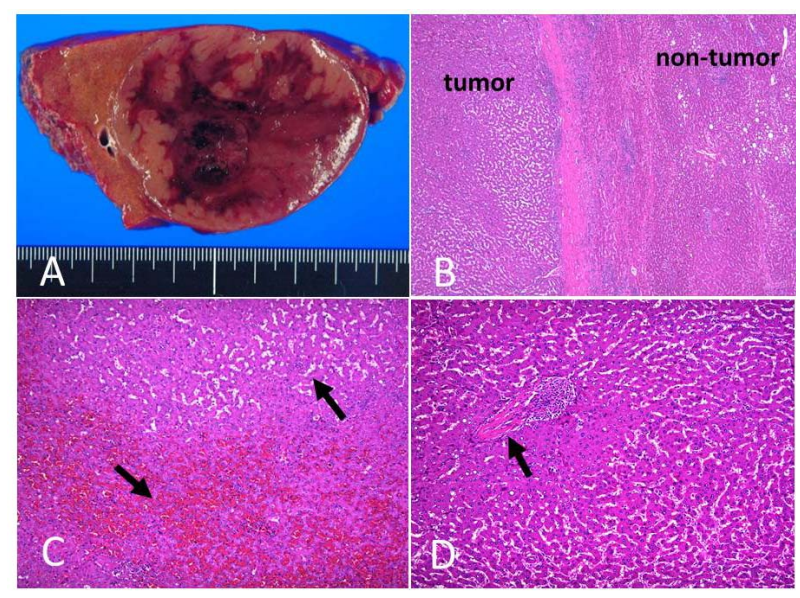

Figure 4. Macroscopic and microscopic findings. (A) The tumor was soft, the cut surface was brown and it was $6 \times 5 \mathrm{~cm}$ in size with a thin capsule. A dark purple congested area was evident at the center of the tumor; (B) It showed trabecular hyperplasia of mildly atypical hepatocytes and increased cell density (H\&E, $\times 40)$; (C) Sinusoidal dilatation (black arrow) and hemorrhage (white arrow) were evident (H\&E, $\times 100)$; (D) Abnormally thick artery (black arrow) and filtration of inflammatory cells (white arrow) were evident $(\mathrm{H} \& \mathrm{E}, \times 100)$.

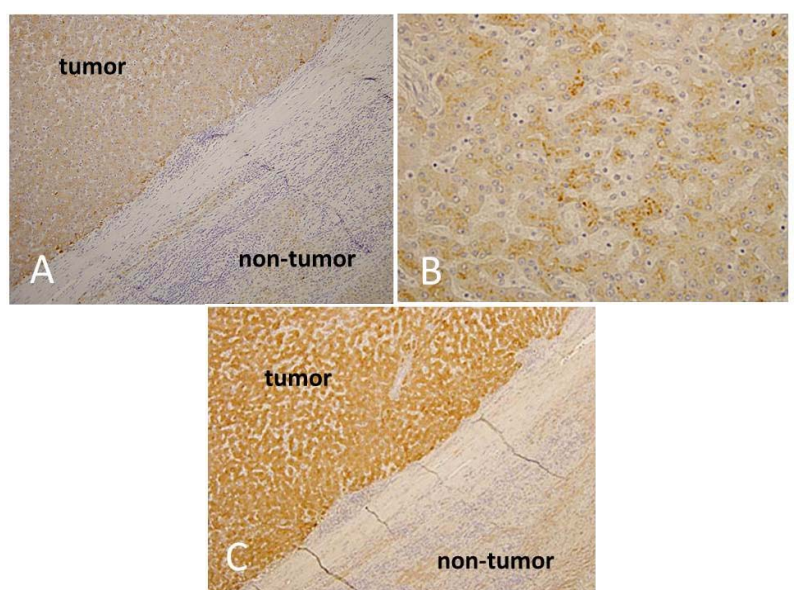

Figure 5. Immunohistological findings. (A) It showed positivity for SAA (SAA, $\times 40$ ); (B) High magnification of SAA staining revealed positivity for SAA in atypical hepatocytes (SAA, $\times 100)$; (C) It showed strong positivity for CRP $(\mathrm{CRP}, \times 40)$.

drogens are well known as the major risk factors for HCAs [3,5]. Classically, about $90 \%$ of HCA cases occur in young women, with low incidences in children, males and the elderly [5]. However, recent studies showed that IHCA have more strong correlation with heavy alcohol intake or obesity $[5,6]$ than classically-known pathogenesis.

On diagnostic imaging, differentiating HNF $1 \alpha$-inactivated HCA from other type of HCA is clinically important. The reason is HNF $1 \alpha$-inactivated HCA seldom progresses to hepatocellular carcinoma, whereas malignant transformation will occur in 5\% - 10\% of cases among $\beta$-catenin-activated HCA and IHCA [7]. Laumonier et al. evaluated the possibility of distinguishing subtypes of HCA using sulfur hexafluoride microbubbles (SonoVue, Bracco, Milan, Italy) CEUS. They retrospectively evaluated 17 cases of IHCA versus 16 cases of HNF $1 \alpha-\mathrm{HCA}$, and concluded that the most valuable criteria for the diagnosis of IHCA were the higher rates of hypervascularity (IHCA $100 \%$ vs. HNF $1 \alpha$-HCA $62.5 \%$, p $<0.01$ ), centripetal filling $(94.1 \%$ vs. $25 \%, \mathrm{p}<0.01)$, delayed washout $(64.7 \%$ vs. $12.5 \%, p<0.01)$ and peripheral rim of sustained enhancement $(64.7 \%$ vs. $0 \%, \mathrm{p}<0.01)$ [8]. There are no previous reports for availability of Sonazoid to perform real-time assessment of vascularity in IHCA. In our case, it seems that the CEUS findings using Sonazoid is quite similar to the previously-reported findings using SonoVue agent. These findings indicate the potential of Sonazoid for use in the differential diagnosis of HCA subtypes.

It was reported that IHCA shows distinctive pathological features. Histologically, it exhibits focal or diffuse inflammation, sinusoidal dilatation, congestion and an area of peliosis, along with numerous thick-walled arteries with ductular reaction [3]. Immunohistologically, 
increased expression of inflammatory proteins (SAA and CRP) is detected in tumor hepatocytes. Our case showed a typical pattern, both histologically and immunohistologically.

A molecular gain-of-function mutation in the IL-6 pathway results in marked inflammation in IHCA. In a recent report, a gain-of-function mutation in glycoprotein 130 , a co-receptor for the IL-6 receptor, was reported in $60 \%$ of IHCAs [4]. A gain-of-function mutation in STAT3 , downstream in the IL-6 pathway, also occurs in $8 \%$ of IHCAs [9]. These mutations play an important role in the pathogenesis of IHCA. In this regard, attention should be paid to the findings of further research.

Regarding the malignant potential of IHCA, Dokmak et al. reported on 122 HCA patients, finding IHCA in 66 patients and malignant transformation in 7 cases $(10.6 \%)$ [10]. They also mentioned that maleness was a risk factor of malignant transformation, and $\geq 5 \mathrm{~cm}$ in diameter was associated with a higher risk of intratumoral hemorrhage [10].We consider that rapid enlargement in our case can be attributed to the intratumoral hemorrhage without transforming to the malignancy.

As another option for treatment, hepatic arterial chemoembolization has also been reported [11]. However, tumor behavior has not been completely elucidated, and so have the concerns about malignant transformation. Morenover, the tumor ruptured cannot be dispelled. We think surgical resection is the most adequate treatment at the present.

\section{REFERENCES}

[1] Bioulac-Sage, P., Rebouissou, S., Thomas, C., Blanc, J.F., Saric, J., Cunha, A.S., Rullier, A., Cubel, G., Couchy, G., Imbeaud, S., et al. (2007) Hepatocellular adenoma subtype classification using molecular markers and immunohistochemistry. Hepatology, 46, 740-748. doi:10.1002/hep.21743

[2] Zucman-Rossi, J., Jeannot, E., Nhieu, J.T., Scoazec, J.Y., Guettier, C., Rebouissou, S., Bacq, Y., Leteurtre, E., Paradis, V., Michalak, S., et al. (2006) Genotype-phenotype correlation in hepatocellular adenoma: New classification and relationship with HCC. Hepatology, 43, 515-524. doi:10.1002/hep. 21068
[3] Bioulac-Sage, P., Balabaud, C. and Wanless, I. (2000) Focal nodular hyperplasia and hepatocellular adenoma. In: Bosman, F., Ed., WHO classification of tumours of the digestive system. 4th Edition, IARC, Lyon, 198-204.

[4] Rebouissou, S., Amessou, M., Couchy, G., Poussin, K., Imbeaud, S., Pilati, C., Izard, T., Balabaud, C., BioulacSage, P. and Zucman-Rossi, J. (2009) Frequent in-frame somatic deletions activate gp130 in inflammatory heaptocellular tumours. Nature, 457, 200-204.

doi:10.1038/nature07475

[5] Sasaki, M., Yoneda, N., Kitamura, S., Sato, Y. and Nakanuma, Y. (2011) Characterization of hepatocellular adenoma based on the phenotypic classification: The Kanazawa experience. Hepatology Research, 41, 982-988. doi:10.1111/j.1872-034X.2011.00851.x

[6] Paradis, V., Champault, A., Ronot, M., Deschamps, L., Valla, D.C., Vidaud, D., Vilgrain, V., Belghiti, J. and Bedossa, P. (2007) Telangiectatic adenoma: An entity associated with invreased body mass index and inflammation. Hepatology, 46, 140-146. doi:10.1002/hep.21684

[7] Katabathina, V.S., Menias, C.O., Shanbhogue, A.K.P., Fagirdar, F., Paspulati, R.M. and Prasad, S.R. (2011) Genetics and imaging of hepatocellular adenomas: 2011 update. AbdGast Imaging, 31, 1529-1543.

[8] Laumonier, H., Cailliez, H., Balabaud, C., Possenti, L., Zucman-Rossi, J., Bioulac-Sage, P. and Trillaud, H. (2012) Role of contrast-enhanced sonography in differentiation of subtypes of hepatocellular adenoma: Correlation with MRI findings. American Journal of Roentgenology, 199, 341-348. doi:10.2214/AJR.11.7046

[9] Pliati, C., Amessou, M., Bihl, M.P., Balabaud, C., Nhieu, J.T., Paradis, V., Nault, J.C., Izard, T., Bioulac-Sage, P., Couchy, G., et al. (2011) Somatic mutations activating STAT3 in human inflammatory hepatocellular adenomas. Journal of Experimental Medicine, 208, 1359-1366. doi:10.1084/jem.20110283

[10] Dokmak, S., Paradis, V., Vilgrain, V., Sauvanet, A., Farges, O., Valla, D., Bedossa, P. and Belghiti, J. (2009) A single-center surgical experience of 122 patients with single and multiple hepatocellular adenomas. Gastroenterology, 137, 1698-1705. doi:10.1053/j.gastro.2009.07.061

[11] Stoot, J.K., van de Linden, E., Terpstra, O.T. and Schaapherder, A.F. (2007) Life-saving therapy for haemorrhaging liver adenomas using selective arterial embolization. British Journal of Surgery, 94, 1249-1253. doi:10.1002/bjs.5779 